九州大学学術情報リポジトリ

Kyushu University Institutional Repository

\title{
TREE EXPRESSIONS AND THEIR PRODUCT FORMULA
}

Inokuchi, Shuichi

Faculty of Mathematics, Kyushu University

Kawahara, Yasuo

Department of Informatics, Kyushu University

https://doi.org/10.5109/13521

出版情報: Bulletin of informatics and cybernetics. 35 (1/2), pp.69-84, 2003-12. Research Association of Statistical Sciences

バージョン :

権利関係 : 


\title{
TREE EXPRESSIONS AND THEIR PRODUCT FORMULA
}

\author{
By \\ Shuichi INoKUCHI* and Yasuo KaWAHARA ${ }^{\dagger}$
}

\begin{abstract}
In this paper we propose tree expressions which represent transition diagrams of finite transition systems by algebraic formulas. We consider the cartesian product of transition systems and present a product formula of tree expressions. By the product formula we easily get the tree expression of the product of transition systems represented by tree expressions.
\end{abstract}

Key Words and Phrases: tree expression, product formula, transition system

\section{Introduction}

Many artificial transition systems have been devised by engineers and scientists. They investigated transition systems and applied them to many fields. Generally transition systems are represented by a pair $(X, f)$ of a non empty set $X$ and a transition function $f$ on $X$. It is not easy to understand behaviors of a transition system $(X, f)$. So in order to understand behaviors of the transition systems more easily we describe their transition diagrams by graphs showing their transition. If the number of $X$ is small then we can easily describe the transition diagram. But if the number of $X$ is large then we cannot describe it.

So we consider special transition systems such that they can be separated or are composed by several transition systems. If a transition system can be separated, then analysing small separated transition systems is a clue to understand global behaviors. Two transition systems have simple behavior but often their combination behave complexly. So we investigate a product of transition systems.

There are several studies on a cartesian product of transition systems. Kumamoto and Nohmi (in preparation) analysed behaviors of integral affine transition systems, and showed that the transition diagrams of integral affine transition systems are the cartesian product of a system whose nodes are all on a limit cycle and a system having a fixed point. Lee and Kawahara (1996) presented tree expressions and their product formula, and got the result that transition diagram of some cellular automata can be represented by the cartesian product of transition diagrams of cellular automata with smaller cells. But their tree expressions can represent only transition systems with a fixed point and their product formula is limited on transition systems such that they have a fixed point

\footnotetext{
* Faculty of Mathematics, Kyushu University 33, Fukuoka 812-8581 Japan. inokuchi@math.kyushuu.ac.jp

† Department of Informatics, Kyushu University 33, Fukuoka 812-8581 Japan. kawahara@i.kyushuu.ac.jp
} 
and their height is less than 2. Namely their product formula can't apply to transition systems having a limit cycle of period length $p$ and more than 3 height.

Cellular automata are the subjects investigated by many researchers and behaviors of cellular automata with some rules are shown. Kawahara (1991), Lee and Kawahara (1992), Kawahara et al. (1995) investigated 1-D and 2-D cellular automata with linear rules 60 and 90, and Inokuchi and Sato (2000) investigated behaviors of 1-D cellular automata with threshold rules and showed that the number of limit cycles and transient lengths.

In this paper we introduce new 'tree expressions' which represent transition diagrams of all finite transition systems in algebraic formulas in order to investigate transition diagrams of transition systems, especially cellular automata. And we investigate the cartesian product of finite transition systems, and present a product formula of tree expressions. The product formula presented in this paper can be applied to all finite transition systems.

\section{Preliminaries}

In the following sections we introduce tree expressions, which are special finite transition systems, and provide their product formulas. So in this section we define necessary notations and state properties of finite systems for after discussion.

A system (or transition system) $(X, f)$ is a pair of a nonempty set $X$ and a transition function $f: X \rightarrow X$. A system $(X, f)$ is finite if $X$ is a finite set. A system $(X, f)$ is connected if for every $x, y \in X$ there exist nonnegative integers $m$ and $n$ such that $f^{m}(x)=f^{n}(y)$. A subsystem $(S, f \mid S)$ of $(X, f)$ is a system such that $S \subset X$ and $(f \mid S)(x)=f(x) \in S$ for every $x \in S$. (That is, the restriction $f \mid S$ of $f$ on $S$ defines a function $S \rightarrow S$.) Usually a subsystem $(S, f \mid S)$ of $(X, f)$ is denoted by $S$ for short.

A dynamorphism $\varphi:(X, f) \rightarrow(Y, g)$ from a system $(X, f)$ into another system $(Y, g)$ is a function $\varphi: X \rightarrow Y$ such that $g \varphi=\varphi f$. A dynamorphism $\varphi:(X, f) \rightarrow(Y, g)$ is called an isomorphism of systems if there exists an inverse dynamorphism $\psi:(Y, g) \rightarrow$ $(X, f)$ such that $\varphi \psi=i d_{Y}$ and $\psi \varphi=i d_{X}$ where $i d_{X}$ and $i d_{Y}$ are identity functions on $X$ and $Y$, respectively. It is trivial that isomorphic systems have isomorphic transition diagrams.

We first show that every finite system can be decomposed into a disjoint union of connected ones.

Proposition 2.1. Every finite system $(X, f)$ is a disjoint union (coproduct) of connected subsystems. That is, $X=X_{1}+\cdots+X_{k}$, where $X_{i}$ is a connected subsystem for $i=1, \cdots, k$.

Let $(X, f)$ be a system. For a nonnegative integer $n n$-th images $f^{n}(X)$ of $f$ is a subset of $X$ such that $x \in f^{n}(X)$ if and only if there exists $z \in X$ with $x=f^{n}(z)$. Note that $f^{n}(X) \neq \emptyset$ for each nonnegative integer $n$ since $X \neq \emptyset$, and the following descending chain condition holds:

$$
X=f^{0}(X) \supseteq f(X) \supseteq \cdots \supseteq f^{n}(X) \supseteq f^{n+1}(X) \supseteq \cdots .
$$

Now we define $f^{\infty}(X)=\bigcap_{n \geq 0} f^{n}(X)$.

LEMMA 2.2. Let $(X, f)$ be a connected finite system. If $x \in f^{\infty}(X)$ and $m=$ $\min \left\{k>0 ; x=f^{k}(x)\right\}$, then $f^{\infty}(X)=\left\{x, f(x), \cdots, f^{m-1}(x)\right\}$ and $\left|f^{\infty}(X)\right|=m$. 
Let $(X, f)$ be a connected finite system, $x \in f^{\infty}(X)$ and $\left|f^{\infty}(X)\right|=m$. Then $<x, f(x), \cdots, f^{m-1}(x)>$ is called a limit cycle of period length $m$ of $(X, f)$. In particular if $m=1$ then $\langle x\rangle$ is called a fixed point.

Let $(X, f)$ be a finite system. For an element $x \in X$ the height $h_{X}(x)$ of $x$ from limit elements $f^{\infty}(X)$ and the root $r_{X}(x)$ below $x$ are defined as follows:

$$
h_{X}(x)=\min \left\{k \geq 0 ; f^{k}(x) \in f^{\infty}(X)\right\}
$$

and

$$
r_{X}(x)=f^{h_{X}(x)}(x) .
$$

Also the height $H(X, f)$ of $(X, f)$ is defined by

$$
H(X, f)=\min \left\{n \geq 0 ; f^{n}(X)=f^{\infty}(X)\right\} .
$$

And for an arbitrary element $x \in X$ we define $f_{*}^{-1}(x), X(x)$ and $X_{*}(x)$ by

$$
\begin{gathered}
f_{*}^{-1}(x)=f^{-1}(x)-f^{\infty}(X), \\
X(x)=f_{*}^{-1}(x)+\bigcup_{u \in f_{*}^{-1}(x)} X(u)
\end{gathered}
$$

and

$$
X_{*}(x)=\{x\}+X(x) .
$$

Namely, $X(x)$ is the set of all $y \in X$ such that $y \notin f^{\infty}(X)$ and $x=f^{k}(y)$ for some positive integer $k$, and $X_{*}(x)$ is the set of all $z \in X$ such that $z \notin f^{\infty}(X)$ and $x=f^{h}(z)$ for some nonnegative integer $h$.

Proposition 2.3. Let $(X, f)$ be a connected system with a limit cycle $\left\langle x_{0}, \cdots, x_{p-1}\right\rangle$ of period length $p$. Then $(X, f)$ is the disjoint union of $X_{*}\left(x_{i}\right)$ for $0 \leq i \leq p-1$. That is,

$$
X=\bigcup_{i=0}^{p-1} X_{*}\left(x_{i}\right) \text { (disjoint union). }
$$

In particular, $X=X_{*}\left(x_{0}\right)$ for any system $X$ with a limit cycle (or fixed point) $\left\langle x_{0}\right\rangle$ of period length 1 .

\section{Tree Expressions}

In this section we define tree expressions which are special systems, and present a function which transforms transition systems into tree expressions.

Let $N$ be the set of all positive integers, $N^{*}$ the set of all finite strings of positive integers including the empty string $\varepsilon$, and $N^{+}$the set of all nonempty strings in $N^{*}$. As in formal language theory we use two operations + (set union) and - (concatenation) on subsets of $N^{*}$, that is, $S_{1}+S_{2}=\left\{w \in N^{*} ; w \in S_{1}\right.$ or $\left.w \in S_{2}\right\}$ and $S_{1} \cdot S_{2}=\left\{w_{1} \cdot w_{2} \in\right.$ $N^{*} ; w_{1} \in S_{1}$ and $\left.w_{2} \in S_{2}\right\}$, where $S_{1}$ and $S_{2}$ are subsets of $N^{*}$. Define a function $\mu: N^{*} \rightarrow N^{*}$ by $\mu(\varepsilon)=\varepsilon$ and $\mu(w \cdot k)=w$ for all $w \in N^{*}$ and $k \in N$. Then $\left(N^{*}, \mu\right)$ is a connected infinite system with a unique fixed point $\varepsilon$. We often write $n$ for a singleton set $\{n\}$. 
Definition 3.1. A p-set (pretree set) is a finite subset of $N^{+}$defined as follows:

1. The empty set is a p-set, denoted by [0].

2. If $m$ is a positive integer and $E_{1}, E_{2}, \cdots, E_{m}$ are p-sets, then

$$
\left[m+\sum_{i=1}^{m} E_{i}\right]=\{1,2, \cdots, m\}+\bigcup_{i=1}^{m} i \cdot E_{i} \text { (disjoint union) }
$$

is a p-set.

A p-set means the information of inputs of a node which is not on a limit cycle. For example,

$$
\begin{aligned}
& {[3+[0]+[0]+[0]]=\{1,2,3\} } \\
& {[3+[2+[0]+[0]]+[0]+[0]] }=\{1,2,3\}+1 \cdot[2+[0]+[0]]+2 \cdot[0]+3 \cdot[0] \\
&=\{1,2,3\}+1 \cdot\{1,2\} \\
&=\{1,2,3,1 \cdot 1,1 \cdot 2\},
\end{aligned}
$$

so they represent Figure 1 and 2.

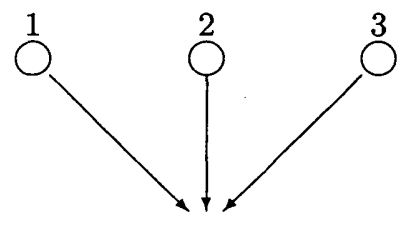

Figure 1: $[3+[0]+[0]+[0]]$

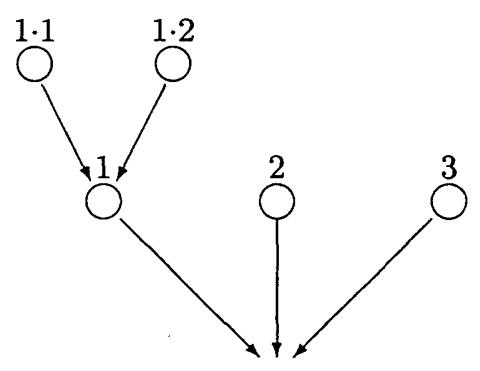

Figure 2: $[3+[2+[0]+[0]]+[0]+[0]]$

Definition 3.2. A t-set (tree set) is a subset of $N^{*}$ recursively defined as follows:

1. The singleton set $\{\varepsilon\}$ is a t-set, denoted by $[1]_{*}$. 
2. If $m$ is a positive integer not less than 2 and $E_{1}, E_{2}, \cdots, E_{m-1}$ are p-sets, then

$$
\left[m+\sum_{i=1}^{m-1} E_{i}\right]_{*}=\{\varepsilon\}+\left[(m-1)+\sum_{i=1}^{m-1} E_{i}\right]
$$

is a t-set.

A t-set means the information of inputs of a node which is on a limit cycle. For example,

$$
\begin{aligned}
{[4+[2+[0]+[0]]+[0]+[0]]_{*} } & =\{\varepsilon\}+[3+[2+[0]+[0]]+[0]+[0]] \\
& =\{\varepsilon, 1,2,3,1 \cdot 1,1 \cdot 2\}
\end{aligned}
$$

so it represents Figure 3.

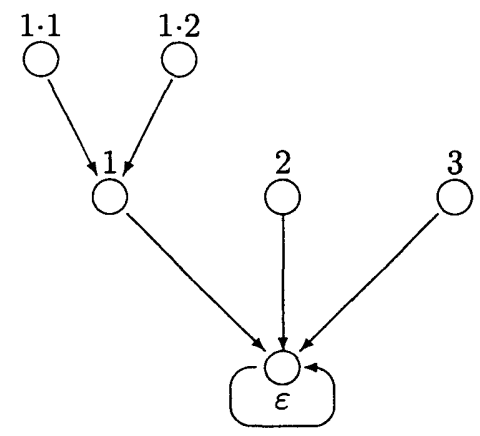

Figure 3: $[4+[2+[0]+[0]]+[0]+[0]]_{*}$

Definition 3.3. Let $T_{0}, T_{1}, \cdots, T_{p-1}$ be t-sets. Then a tree expression

$$
<T_{0}, T_{1}, \cdots, T_{p-1}>=\bigcup_{i=0}^{p-1} i \cdot T_{i}
$$

is a subsystem of $\left(Z(p) N^{*}, \mu_{p}\right)$, where

- $\mu_{p}(i)=i+1(\bmod p)$, and

- $\mu_{p}(i w)=i \cdot \mu(w)$

for $i \in Z(p)$ and $w \in T_{i}^{+}$.

For example,

$$
\begin{aligned}
& <[3+[0]+[2+[0]+[0]]]_{*},[4+[3+[0]+[0]+[0]]+[0]+[2+[0]+[0]]]_{*}> \\
= & 0 \cdot[3+[0]+[2+[0]+[0]]]_{*}+1 \cdot[4+[3+[0]+[0]+[0]]+[0]+[2+[0]+[0]]]_{*} \\
= & 0 \cdot\{\varepsilon, 1,2,2 \cdot 1,2 \cdot 2\}+1 \cdot\{\varepsilon, 1,2,3,1 \cdot 1,1 \cdot 2,1 \cdot 3,3 \cdot 1,3 \cdot 2\} \\
= & \{0,0 \cdot 1,0 \cdot 2,0 \cdot 2 \cdot 1,0 \cdot 2 \cdot 2, \\
& \quad 1,1 \cdot 1,1 \cdot 2,1 \cdot 3,1 \cdot 1 \cdot 1,1 \cdot 1 \cdot 2,1 \cdot 1 \cdot 3,1 \cdot 3 \cdot 1,1 \cdot 3 \cdot 2\}
\end{aligned}
$$




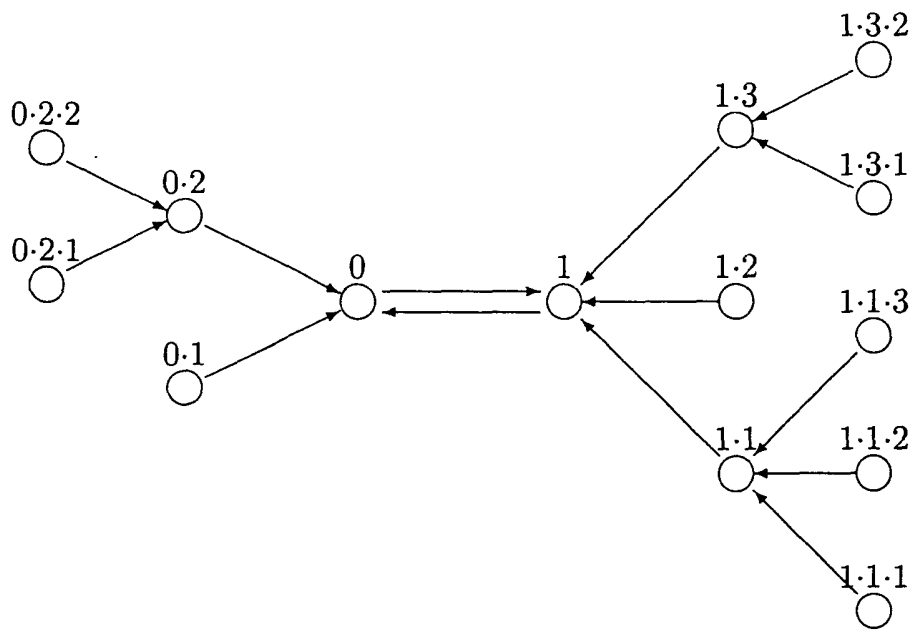

Figure 4: $<\left[3+[0]+[2+[0]+[0]]_{*},[4+[3+[0]+[0]+[0]]+[0]+[2+[0]+[0]]]_{*}>\right.$

so this tree expression represents Figure 4.

Let $E$ be a t-set. Trivially tree expression $\langle E\rangle$ with a fixed point and t-set $E$ are isomorphic. So we often write $E$ for $\langle E\rangle$.

The number of nodes of a system is larger and larger, and the redundancies of its tree expression increase. So in order to reduce the redundancies we introduce notations as follows;

- If $E_{j+1}=E_{j+2}=\cdots=E_{j+n}=[0]$, then a p-set $\left[m+\sum_{i=1}^{m} E_{i}\right]$ and a t-set $\left[m+\sum_{i=1}^{m-1} E_{i}\right]_{*}$ are denoted by $\left[m+\sum_{i=1}^{j} E_{i}+\sum_{i=j+n+1}^{m} E_{i}\right]$ and $\left[m+\sum_{i=1}^{j} E_{i}+\right.$ $\left.\sum_{i=j+n+1}^{m-1} E_{i}\right]_{*}$ respectively.

- If $E_{j+1}=E_{j+2}=\cdots=E_{j+n}$, then a p-set $\left[m+\sum_{i=1}^{m} E_{i}\right]$ and a t-set $[m+$ $\left.\sum_{i=1}^{m-1} E_{i}\right]_{*}$ are denoted by $\left[m+\sum_{i=1}^{j} E_{i}+n E_{j+1}+\sum_{i=j+n+1}^{m} E_{i}\right]$ and $[m+$ $\left.\sum_{i=1}^{j} E_{i}+n E_{j+1}+\sum_{i=j+n+1}^{m-1} E_{i}\right]_{*}$ respectively.

Two tree expressions $<[2+[2]]_{*},[3]_{*},[4+[2]]_{*}>$ and $<[2+[2]]_{*},[4+[2]]_{*},[3]_{*}>$ represent Figure 5 and 6 , and are not isomorphic trivially.

Generally a tree expression $\left\langle E_{0}, E_{1}, \cdots, E_{p-1}\right\rangle$ is not isomorphic to

$<E_{\tau(0)}, E_{\tau(1)}, \cdots, E_{\tau(p-1)}>$, where $\tau$ is a permutation, except for some cases, for example $<E_{p-1}, E_{0}, E_{1}, \cdots, E_{p-2}>$ and so on.

DEFINITION 3.4. An equivalence relation $\sim$ on the set of all p-sets is defined as follows;

1. $[0] \sim[0]$,

2. $\left[m+\sum_{i=1}^{m} E_{i}\right] \sim\left[n+\sum_{j=1}^{n} F_{j}\right]$ if $m=n$ and there is a permutation $\tau$ on the set $\{1,2, \cdots, m\}$ such that $E_{i} \sim F_{\tau(i)}$ for each $i=1,2, \cdots, m$. 


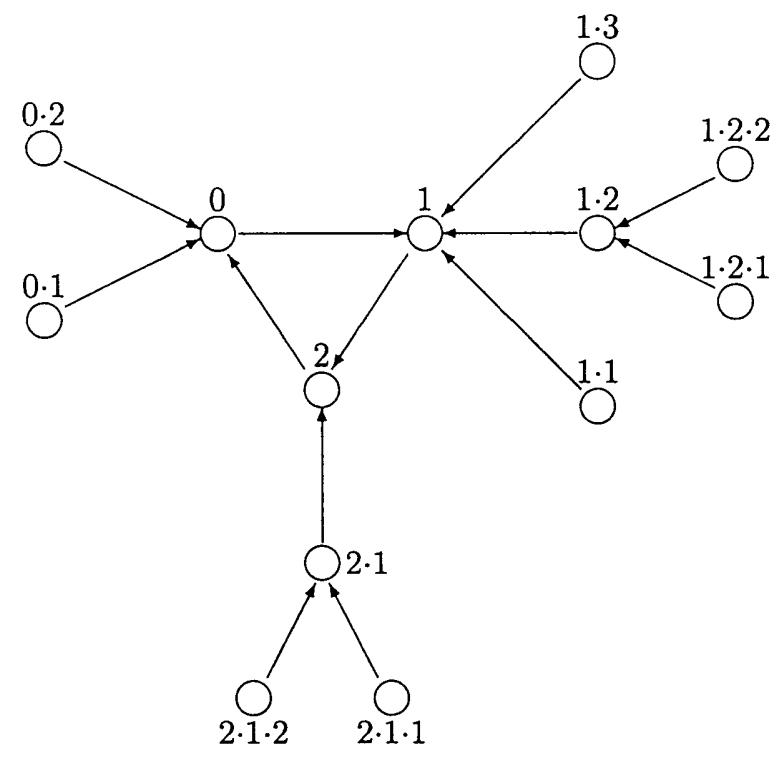

Figure $5:<[2+[2]]_{*},[3]_{*},[4+[2]]_{*}>$

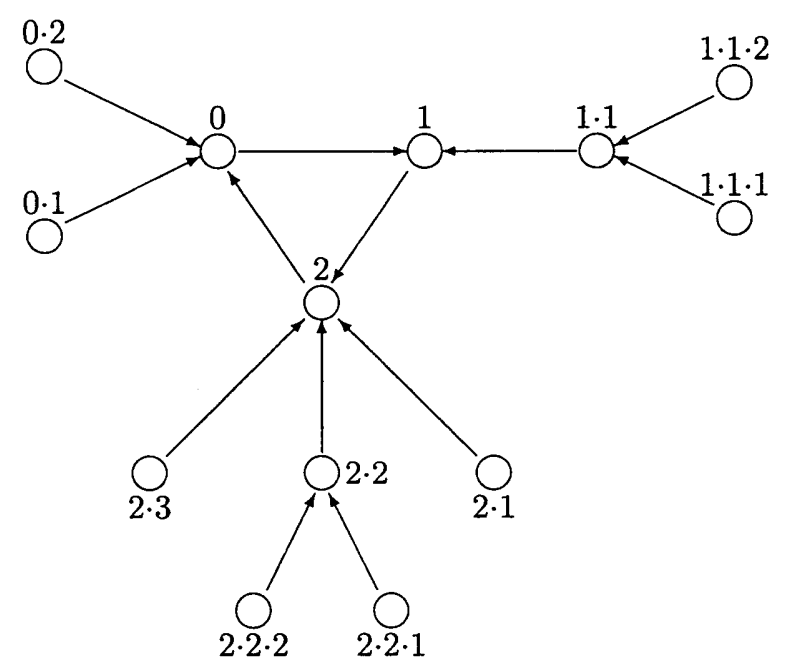

Figure 6: $<[2+[2]]_{*},[4+[2]]_{*},[3]_{*}>$

Definition 3.5. Define $T^{+}=T \cap N^{+}$for a t-set $T$. Note that $T^{+}=[(m-1)+$ $\left.\sum_{i=1}^{m-1} E_{i}\right]$ (a p-set) when $T=\left[m+\sum_{i=1}^{m-1} E_{i}\right]_{*}$. Two t-sets $T$ and $T^{\prime}$ are equivalent if two p-sets $T^{+}$and $T^{\prime+}$ are equivalent.

Proposition 3.6. Let $E$ be a p-set and $T$ a t-set. Then the following hold;

1. If $w \in E$ and $|w|>1$, then $\mu(w) \in E$. 
2. For two equivalent $p$-sets $E$ and $F$ there is a bijection $f: E \rightarrow F$ such that $|f(w)|=|w|$ for all $w \in E$ and $\mu(f(w))=f(\mu(w))$ for all $w \in E$ with $|w|>1$.

3. If $w \in T$, then $\mu(w) \in T$ (that is, $T$ is a finite subsystem of $\left(N^{*}, \mu\right)$ ),

4. Two equivalent $t$-sets are isomorphic as systems. (That is, $T \sim T^{\prime}$ implies $T \cong T^{\prime}$.)

Proof. 1. Let $E=\left[m+\sum_{i=1}^{m} E_{i}\right](m>0)$ be a p-set and $w \in E$ with $|w|>1$. Then $w=i w^{\prime}$ for some integer $i$ such that $1 \leq i \leq m$ and $w^{\prime} \in E_{i}$. If $\left|w^{\prime}\right|=1$, then $\mu(w)=i \in\{1,2, \cdots, m\} \subseteq E$. If $\left|w^{\prime}\right|>1$, then $\mu\left(w^{\prime}\right) \in E_{i}$ by the induction hypothesis. So $\mu(w)=i \mu\left(w^{\prime}\right) \in i E_{i} \subseteq E$.

2. Let $E=\left[m+\sum_{i=1}^{m} E_{i}\right]$ and $F=\left[m+\sum_{i p=1}^{m} F_{i}\right](m>0)$ be equivalent p-sets and $\tau$ a permutation such that $E_{i}=F_{\tau(i)}$. Assume that $f_{i}: E_{i} \rightarrow F_{\tau(i)}$ is a bijection $(i=1, \cdots, m)$ such that $\mu\left(f_{i}\left(w_{i}\right)\right)=f_{i}\left(\mu\left(w_{i}\right)\right)$ for all $w_{i} \in E_{i}$ such that $\left|w_{i}\right|>1$. Define $f: E \rightarrow F$ as follows: $f(\varepsilon)=\varepsilon, f(i)=\tau(i)$ and $f\left(i w_{i}\right)=\tau(i) f_{i}\left(w_{i}\right)$ for $i=1, \cdots, m$ and $w_{i} \in E_{i}$. Finally it is easy to check that $f: E \rightarrow F$ is a desired bijection.

3. It is trivial from 1.

4. It immediately follows from 2 .

By tree expressions defined above we can represent the transition diagrams of arbitrary finite systems. We define a transformation function $\gamma$ to get tree expressions of finite systems as follows;

Definition 3.7. Let $(X, f)$ be a connected finite system with a limit cycle $<x_{0}, \cdots, x_{p-1}>$ of period length $\mathrm{p}$. Then a transformation function $\gamma$ is defined as follows;

$$
\begin{gathered}
\gamma(X)=<\gamma\left(X_{*}\left(x_{0}\right)\right), \gamma\left(X_{*}\left(x_{1}\right)\right), \cdots, \gamma\left(X_{*}\left(x_{p-1}\right)\right)> \\
\gamma\left(X_{*}(x)\right)=\left[\left|f_{*}^{-1}(x)\right|+1+\sum_{u \in f_{*}^{-1}(x)} \gamma(X(u))\right]_{*}
\end{gathered}
$$

and

$$
\left.\gamma(X(u))=|| f_{*}^{-1}(u) \mid+\sum_{u^{\prime} \in f^{-1}(u)} \gamma\left(X\left(u^{\prime}\right)\right)\right] .
$$

And we show that the transformation function $\gamma$ defined above is an isomorphism, that is, $\left(\gamma(X), \mu_{p}\right) \cong(X, f)$.

THEOREM 3.8. Let $(X, f)$ be a connected finite system with a limit cycle of period length $p$. Then the system $\left(\gamma(X), \mu_{p}\right)$ is isomorphic to $(X, f)$, that is, $\left(\gamma(X), \mu_{p}\right) \cong$ $(X, f)$.

Proof. Let $(X, f)$ be a connected finite system with a limit cycle $<x_{0}, x_{1}, \cdots, x_{p-1}>$ of period length $p$. 
1. In the case that $H(X, f)=0$, that is, $(X, f)$ is a system such that $X=\left\{x_{0}, x_{1}, \cdots, x_{p-1}\right\}$ and $f\left(x_{i}\right)=x_{i+1}(\bmod p)$. Then we have

$$
\begin{aligned}
X_{*}\left(x_{i}\right) & =\left\{x_{i}\right\}+f_{*}^{-1}\left(x_{i}\right)+\bigcup_{u \in f_{*}^{-1}\left(x_{i}\right)} X(u) \\
& =\left\{x_{i}\right\}
\end{aligned}
$$

for any $i(0 \geq i \geq p-1)$. We have

$$
\begin{aligned}
\gamma(X) & =\left\langle\gamma\left(X_{*}\left(x_{0}\right)\right), \gamma\left(X_{*}\left(x_{1}\right)\right), \cdots, \gamma\left(X_{*}\left(x_{p-1}\right)\right),>\right. \\
& =<[1]_{*},[1]_{*}, \cdots,[1]_{*}> \\
& =\bigcup_{i=0}^{p-1} i \cdot[1]_{*} \\
& =\{0,1, \cdots, p-1\}
\end{aligned}
$$

Therefore we have $(X, f) \cong\left(\gamma(X), \mu_{p}\right)$.

2. In the case that $H(X, f)=1$.

Then $X_{*}\left(x_{i}\right)=\left\{x_{i}\right\}+f_{*}^{-1}\left(x_{i}\right)$. So we have

$$
\begin{aligned}
\gamma(X) & =<\gamma\left(X_{*}\left(x_{0}\right)\right), \gamma\left(X_{*}\left(x_{1}\right)\right), \cdots, \gamma\left(X_{*}\left(x_{p-1}\right)\right)> \\
& =\bigcup_{i=0}^{p-1} i \cdot \gamma\left(X_{*}\left(x_{i}\right)\right. \\
& =\bigcup_{i=0}^{p-1} i \cdot\left[\left|f_{*}^{-1}\left(x_{i}\right)\right|+1+\left|f_{*}^{-1}\left(x_{i}\right)\right|[0]\right]_{*} \\
& =\bigcup_{i=0}^{p-1} i+\bigcup_{i=0}^{p-1} i \cdot\left[\left|f_{*}^{-1}\left(x_{i}\right)\right|+\left|f_{*}^{-1}\left(x_{i}\right)\right|[0]\right] .
\end{aligned}
$$

From (1) we have

$$
\left(f^{\infty}(X), f \mid f^{\infty}(X)\right) \cong\left(\{0,1, \cdots, p-1\}, \mu_{p} \mid\{0,1, \cdots, p-1\}\right) .
$$

Now we show

$$
\begin{aligned}
& \left(f_{*}^{-1}\left(x_{i}\right), f \mid f_{*}^{-1}\left(x_{i}\right)\right) \\
\cong & \left(i \cdot\left[\left|f_{*}^{-1}\left(x_{i}\right)\right|+\left|f_{*}^{-1}\left(x_{i}\right)\right|[0]\right], \mu_{p} \mid i \cdot\left[\left|f_{*}^{-1}\left(x_{i}\right)\right|+\left|f_{*}^{-1}\left(x_{i}\right)\right|[0]\right]\right)
\end{aligned}
$$

for any $i(0 \geq i \geq p-1)$. It is clear that

$$
\left|f_{*}^{-1}\left(x_{i}\right)\right|=\left|i \cdot\left[\left|f_{*}^{-1}\left(x_{i}\right)\right|+\left|f_{*}^{-1}\left(x_{i}\right)\right|[0]\right]\right| .
$$

$f \mid f_{*}^{-1}\left(x_{i}\right)(x)$ is undefined for any $x \in f_{*}^{-1}\left(x_{i}\right)$ and $\mu_{p} \mid i \cdot\left[\left|f_{*}^{-1}\left(x_{i}\right)\right|+\left|f_{*}^{-1}\left(x_{i}\right)\right|[0]\right](y)$ is undefined for any $y \in i \cdot\left[\left|f_{*}^{-1}\left(x_{i}\right)\right|+\left|f_{*}^{-1}\left(x_{i}\right)\right|[0]\right]$. Thus we have

$$
\begin{aligned}
& \left(f_{*}^{-1}\left(x_{i}\right), f \mid f_{*}^{-1}\left(x_{i}\right)\right) \\
\cong & \left(i \cdot\left[\left|f_{*}^{-1}\left(x_{i}\right)\right|+\left|f_{*}^{-1}\left(x_{i}\right)\right|[0]\right], \mu_{p} \mid i \cdot\left[\left|f_{*}^{-1}\left(x_{i}\right)\right|+\left|f_{*}^{-1}\left(x_{i}\right)\right|[0]\right]\right) .
\end{aligned}
$$


Now we let $k$ and $h_{i}$ be an isomorphism from $f^{\infty}(X)$ to $\{0,1, \cdots, p-1\}$ and from $f_{*}^{-1}\left(x_{i}\right)$ to $i \cdot\left[\left|f_{*}^{-1}\left(x_{i}\right)\right|+\left|f_{*}^{-1}\left(x_{i}\right)\right|[0]\right]$, respectively. We define the function $h: X \longrightarrow \gamma(X)$ as follows;

$$
h(x)=\left\{\begin{array}{lll}
k(x) & \text { if } & x \in f^{\infty}(X) \\
k\left(x_{i}\right) \cdot h_{i}(x) & \text { if } & x \in f_{*}^{-1}\left(x_{i}\right)
\end{array}\right.
$$

We show that the function $h$ is an isomorphism. First we assume that $x \in f^{\infty}(X)$. Then we have

$$
\begin{aligned}
h f(x) & =h(f(x)) \\
& =k(f(x)) \\
& =\mu_{p}(k(x)) \\
& =\mu_{p}(h(x)) \\
& =\mu_{p} h(x)
\end{aligned}
$$

Next we assume that $x \in f_{*}^{-1}\left(x_{i}\right)$. Then we have

$$
\begin{aligned}
h f(x) & =h\left(x_{i}\right) \\
& =k\left(x_{i}\right) \\
& =\mu_{p}\left(k\left(x_{i}\right) h_{i}(x)\right) \\
& =\mu_{p} h(x)
\end{aligned}
$$

Thus the function $h$ is an isomorphism. So we have $(X, f) \cong\left(\gamma(X), \mu_{p}\right)$.

3. In the case that $H(X, f) \geq 2$.

First we show that the system $(X(x), f \mid X(x))$ is isomorphic to $(\gamma(X(x)), \mu \mid \gamma(X(x)))$ for any $x \in X-f^{-1}\left(f^{\infty}(X)\right)$ by induction. Let $x$ be in $X-f^{-1}\left(f^{\infty}(X)\right)$.

(a) Assume that $f^{-1}(x)=\emptyset$.

Then we have $X(x)=\emptyset$ and $\gamma(X(x))=[0]=\emptyset$. So

$$
(X(x), f \mid X(x)) \cong(\gamma(X(x)), \mu \mid \gamma(X(x)))
$$

(b) Assume that $f^{-1}(x) \neq \emptyset$ and $f^{-2}(x)=f^{-1}\left(f^{-1}(x)\right)=\emptyset$.

Then $X(x)=f_{*}^{-1}(x)+\bigcup_{u \in f_{*}^{-1}(x)} X(u)$ and $f \mid X(x)(y)$ is undefined for any $y \in X(x)$. And we have

$$
\begin{aligned}
\gamma(X(x)) & =\left[\left|f_{*}^{-1}(x)\right|+\left|f_{*}^{-1}(x)\right|[0]\right] \\
& =\left\{0,1, \cdots,\left|f_{*}^{-1}(x)\right|-1\right\}
\end{aligned}
$$

and $\mu \mid \gamma(X(x))(z)$ is undefined for any $z \in \gamma(X(x))$. So

$$
(X(x), f \mid X(x)) \cong(\gamma(X(x)), \mu \mid \gamma(X(x)))
$$


(c) Assume that $(X(y), f \mid X(y)) \cong(\gamma(X(y)), \mu \mid \gamma(X(y)))$ for any $y \in X-f^{-1}\left(f^{\infty}(X)\right)$ such that $f_{*}^{-n+1}(y)=\emptyset$ and $f_{*}^{-n+1}(x) \neq \emptyset$ and $f_{*}^{-n}(x)=\emptyset$.

Then

$$
X(x)=f_{*}^{-1}(x)+\bigcup_{u \in f_{*}^{-1}(x)} X(u)
$$

and

$$
\gamma(X(x))=\left[\left|f_{*}^{-1}(x)\right|+\sum_{u \in f_{*}^{-1}(x)} \gamma(X(u))\right] .
$$

By the induction hypothesis we have

$$
(X(u), f \mid X(u)) \cong(\gamma(X(u)), \mu \mid \gamma(X(u)))
$$

for any $u \in f_{*}^{-1}(x)$. Now we let the function $h_{u}: X(u) \rightarrow \gamma(X(u))$ be an isomorphism, and define the function $h: X(x) \rightarrow \gamma(X(x))$ as follows;

$$
h(y)=\left\{\begin{array}{lll}
s(y) & \text { if } \quad y \in f_{*}^{-1}(x) \\
s(u) \cdot h_{u}(y) & \text { if } \quad y \in X(u) \text { and } u \in f_{*}^{-1}(x)
\end{array}\right.
$$

where $s: f_{*}^{-1}(x) \rightarrow N$ is a set function. It is an isomorphism. Thus This derive

$$
(X(x), f \mid X(x)) \cong(\gamma(X(x)), \mu \mid \gamma(X(x)))
$$

for any $x \in X-f_{*}^{-1}\left(f^{\infty}(X)\right)$.

From (2)

$$
\begin{aligned}
& \left(f^{-1}\left(f^{\infty}(X)\right), f \mid f^{-1}\left(f^{\infty}(X)\right)\right) \\
\cong & \left(\gamma\left(f^{-1}\left(f^{\infty}(X)\right)\right), \mu_{p} \mid \gamma\left(f^{-1}\left(f^{\infty}(X)\right)\right)\right),
\end{aligned}
$$

and so we let $k$ be an isomorphism. We define the function $g: X \rightarrow \gamma(X)$ as follows;

$$
g(x)=\left\{\begin{array}{lll}
k(x) & \text { if } & x \in f^{-1}\left(f^{\infty}(X)\right) \\
k(u) \cdot k_{u}(x) & \text { if } & x \in X(u) \text { and } u \in f^{-1}\left(f^{\infty}(X)\right)
\end{array}\right.
$$

where $k_{u}: X(u) \longrightarrow \gamma(X(u))$ is isomorphism for $u \in f^{-1}\left(f^{\infty}(X)\right)$. Then the function $g$ is an isomorphism.

\section{Products of Systems and Product Formula}

In this section we discuss the product of connected finite systems, and present a product formula of tree expressions.

Let $X=(X, f)$ and $Y=(Y, g)$ be two systems. The (cartesian) product $X \times Y$ of $X$ and $Y$ is a system

$$
(X \times Y, f \times g)
$$

where $(f \times g)(x, y)=(f(x), g(y))$ for $x \in X$ and $y \in Y$.

Kumamoto and Nohmi (in preparation) analysed the transition diagram of integral affine transition systems and decided the transition diagram of any integral affine 
transition systems with the cartesian product. And they presented the product formula of transition diagrams of integral affine transition systems.

From now we discuss the cartesian product of connected finite systems and present a product formula of tree expressions.

Let $X=(X, f)$ and $Y=(Y, g)$ be two systems, and $\left\langle x_{0}, \cdots, x_{p-1}\right\rangle$ and $<$ $y_{0}, \cdots, y_{q-1}>$ be two limit cycles of $X$ and $Y$ with period length $p$ and $q$, respectively. Then $X \times Y$ have $\operatorname{gcd}(p, q)$ limit cycles of period length $l c m(p, q)$, where $\operatorname{gcd}(p, q)$ and $\operatorname{lcm}(p, q)$ are the greatest common divisor and the least common multiple of $p$ and $q$, respectively. Limit cycles of $X \times Y$ can be known if limit cycles of $\mathrm{X}$ and $\mathrm{Y}$ are known. So applying of transformation function $\gamma$ to $X \times Y$ we have its isomorphic tree expression.

Note that

$$
\left.(f \times g)_{*}^{-1}\left(x_{i}, y_{j}\right)=f_{*}^{-1}\left(x_{i}\right) \times g_{*}^{-1}\left(y_{j}\right)+f_{*}^{-1}\left(x_{i}\right) \times\left\{y_{j-1}\right\}\right)+\left\{x_{i-1}\right\} \times g_{*}^{-1}\left(y_{j}\right) .
$$

Then we have

$$
\begin{aligned}
& \gamma\left((X \times Y)_{*}\left(x_{i}, y_{j}\right)\right) \\
= & \left.\|(f \times g)^{-1}\left(x_{i}, y_{j}\right) \mid+\sum_{(u, v) \in(f \times g)_{*}^{-1}\left(x_{i}, y_{j}\right)} \gamma((X \times Y)(u, v))\right]_{*} \\
= & {\left[\left|f^{-1}\left(x_{i}\right) \| g^{-1}\left(y_{j}\right)\right|+\sum_{(u, v) \in f_{*}^{-1}\left(x_{i}\right) \times g_{*}^{-1}\left(y_{j}\right)} X(u) \otimes Y(v)\right.} \\
& \left.+\sum_{u \in f_{*}^{-1}\left(x_{i}\right)} X(u) \otimes Y\left(y_{j-1}\right)+\sum_{v \in g_{*}^{-1}\left(y_{j}\right)} X\left(x_{i-1}\right) \otimes Y(v)\right]_{*},
\end{aligned}
$$

where $X(u) \otimes Y(v)=\gamma((X \times Y)(u, v))$.

For all $(u, v) \in f_{*}^{-1}(x) \times g_{*}^{-1}(y)$

$$
X(u) \otimes Y(v)=\left[\left|f^{-1}(u) \| g^{-1}(v)\right|+\sum_{\left(u^{\prime}, v^{\prime}\right) \in f^{-1}(u) \times g^{-1}(v)} X\left(u^{\prime}\right) \otimes Y\left(v^{\prime}\right)\right] .
$$

For all $u \in f_{*}^{-1}(x)$ we have

$$
(f \times g)_{*}^{-1}\left(u, y_{j-1}\right)=f^{-1}(u) \times g_{*}^{-1}\left(y_{j-1}\right)+f^{-1}(u) \times\left\{y_{j-2}\right\},
$$

so

$$
\begin{aligned}
& X(u) \otimes Y\left(y_{j-1}\right) \\
= & \left.\left\|f^{-1}(u)\right\| g^{-1}\left(y_{j-1}\right) \mid+\sum_{\left(u^{\prime}, v^{\prime}\right) \in(f \times g)_{*}^{-1}\left(u, y_{j-1}\right)} X\left(u^{\prime}\right) \otimes Y\left(v^{\prime}\right)\right] \\
= & \left\|f^{-1}(u)\right\| g^{-1}\left(y_{j-1}\right) \mid+\sum_{\left(u^{\prime}, v^{\prime}\right) \in f_{*}^{-1}(u) \times g_{*}^{-1}\left(y_{j-1}\right)} X\left(u^{\prime}\right) \otimes Y\left(v^{\prime}\right) \\
& \left.+\sum_{u^{\prime} \in f^{-1}(u)} X\left(u^{\prime}\right) \otimes Y\left(y_{j-2}\right)\right] .
\end{aligned}
$$


Similarly, for all $v \in g_{*}^{-1}(y)$ we have

$$
\begin{aligned}
& X\left(x_{i-1}\right) \otimes Y(v) \\
= & \left\|f^{-1}\left(x_{i-1}\right)\right\| g^{-1}(v) \mid+\sum_{\left(u^{\prime}, v^{\prime}\right) \in f_{*}^{-1}\left(x_{i-1}\right) \times g^{-1}(v)} X\left(u^{\prime}\right) \otimes Y\left(v^{\prime}\right) \\
& \left.+\sum_{v^{\prime} \in g^{-1}(v)} X\left(x_{i-2}\right) \otimes Y\left(v^{\prime}\right)\right] .
\end{aligned}
$$

From above discussion and the transformation function $\gamma$ the following formula can be found out.

Theorem 4.1 Product Formula. Let $E=<E_{0}, E_{1}, \cdots, E_{p-1}>$ and $D=<$ $D_{0}, D_{1}, \cdots, D_{q-1}>$ be two tree expressions with limit cycles $\langle 0,1, \cdots, p-1\rangle$ and $<0,1, \cdots, q-1>$ respectively where $E_{i}=\left[m_{i}+E_{i}^{1}+\cdots+E_{i}^{m_{i}-1}\right]_{*}$ and $D_{j}=\left[n_{j}+\right.$ $\left.D_{j}^{1}+\cdots D_{j}^{n_{i}-1}\right]_{*}$. Then a subtree $(E \times D)(i, j)$ of the cartesian product $E \times D$ such that its root is $(i, j)$ is shown recursively as follows:

$$
\begin{aligned}
& (E \times D)(i, j) \\
= & {\left[m_{i} n_{j}+\sum_{k=1}^{m_{i}-1} \sum_{h=1}^{n_{j}-1} E_{i}^{k} \otimes D_{j}^{h}+\sum_{k=1}^{m_{i}-1} E_{i}^{k} \otimes D_{j-1 \bmod q}+\sum_{h=1}^{n_{j}-1} E_{i-1 \bmod p} \otimes D_{j}^{h}\right]_{*} }
\end{aligned}
$$

where

$$
\begin{aligned}
{\left[m+\sum_{i=1}^{m} G_{i}\right] \otimes\left[n+\sum_{j=1}^{n} F_{j}\right] } & =\left[m n+\sum_{i=1}^{m} \sum_{j=1}^{n} G_{i} \otimes F_{j}\right] \\
{\left[m+\sum_{i=1}^{m} F_{i}\right] \otimes D_{j} } & =\left[m n_{j}+\sum_{k=1}^{m} \sum_{h=1}^{n_{j}-1} F_{k} \otimes D_{j}^{h}+\sum_{k=1}^{m} F_{k} \otimes D_{j-1 \bmod q}\right] \\
E_{i} \otimes\left[n+\sum_{j=1}^{n} F_{j}\right] & =\left[m_{i} n+\sum_{k=1}^{m_{i}-1} \sum_{h=1}^{n} E_{i}^{k} \otimes F_{h}+\sum_{h=1}^{n} E_{i-1 \bmod p} \otimes F_{h}\right] .
\end{aligned}
$$

COROLlaRY 4.2. For the product of tree expressions with a fixed point the following formula is acquired;

$$
\begin{aligned}
& \left.<\left[m+\sum_{i=1}^{m-1} E_{i}\right]_{*}>\times<n+\sum_{j=1}^{n-1} F_{j}\right]_{*}> \\
= & <\left[m n+\sum_{i=1}^{m-1} \sum_{j=1}^{n-1} E_{i} \otimes F_{j}+\sum_{i=1}^{m-1} E_{i} \otimes\left[n+\sum_{j=1}^{n-1} F_{j}\right]_{*}+\sum_{j=1}^{n-1}\left[m+\sum_{i=1}^{m-1} E_{i}\right]_{*} \otimes F_{j}\right]_{*}>
\end{aligned}
$$

where

$$
\left[m+\sum_{i=1}^{m} E_{i}\right] \otimes\left[n+\sum_{j=1}^{n} F_{j}\right]=\left[m n+\sum_{i=1}^{m} \sum_{j=1}^{n} E_{i} \otimes F_{j}\right],
$$




$$
\begin{aligned}
{\left[m+\sum_{i=1}^{m} E_{i}\right] \otimes\left[n+\sum_{j=1}^{n-1} F_{j}\right]_{*} } & =\left[m n+\sum_{i=1}^{m} E_{i} \otimes\left[n+\sum_{j=1}^{n-1} F_{j}\right]_{*}+\sum_{i=1}^{m} \sum_{j=1}^{n-1} E_{i} \otimes F_{j}\right] \\
{\left[m+\sum_{i=1}^{m-1} E_{i}\right]_{*} \otimes\left[n+\sum_{j=1}^{n} F_{j}\right] } & =\left[m n+\sum_{j=1}^{n}\left[m+\sum_{i=1}^{m-1} E_{i}\right]_{*} \otimes F_{j}+\sum_{i=1}^{m-1} \sum_{j=1}^{n} E_{i} \otimes F_{j}\right] .
\end{aligned}
$$

Now we give two examples of the product of tree expressions.

First we consider the product of simple tree expressions $<[2+[1+[1+[1]]]]_{*}>$ and $<[2+[1+[1]]]_{*}>$ with a fixed point. The product of these are calculated by using the product formula (Corollary 4.2) and represented by the following tree expression.

$$
\begin{aligned}
& <[2+[1+[1+[1]]]]_{*}>\times<[2+[1+[1]]]_{*}> \\
= & <\left[4+[1+[1+[1]]] \otimes[1+[1]]+[1+[1+[1]]] \otimes[2+[1+[1]]]_{*}\right. \\
& \left.+[2+[1+[1+[1]]]]_{*} \otimes[1+[1]]\right]_{*}> \\
= & <\left[4+[1+[1+[1]] \otimes[1]]+\left[2+[1+[1]] \otimes[2+[1+[1]]]_{*}+[1+[1]] \otimes[1+[1]]\right]\right. \\
& \left.+\left[2+[2+[1+[1+[1]]]]_{*} \otimes[1]+[1+[1+[1]]] \otimes[1]\right]\right]_{*}> \\
= & <\left[4+[1+[1]]+\left[2+\left[2+[1] \otimes[2+[1+[1]]]_{*}+[1] \otimes[1+[1]]\right]+[1+[1]]\right]\right. \\
& +[2+[2]+[1]]]_{*}> \\
= & <[4+[1+[1]]+[2+[2+[2]+[1]]+[1+[1]]]+[2+[2]+[1]]]_{*}>
\end{aligned}
$$

The product of these systems is illustrated as Figure 7.
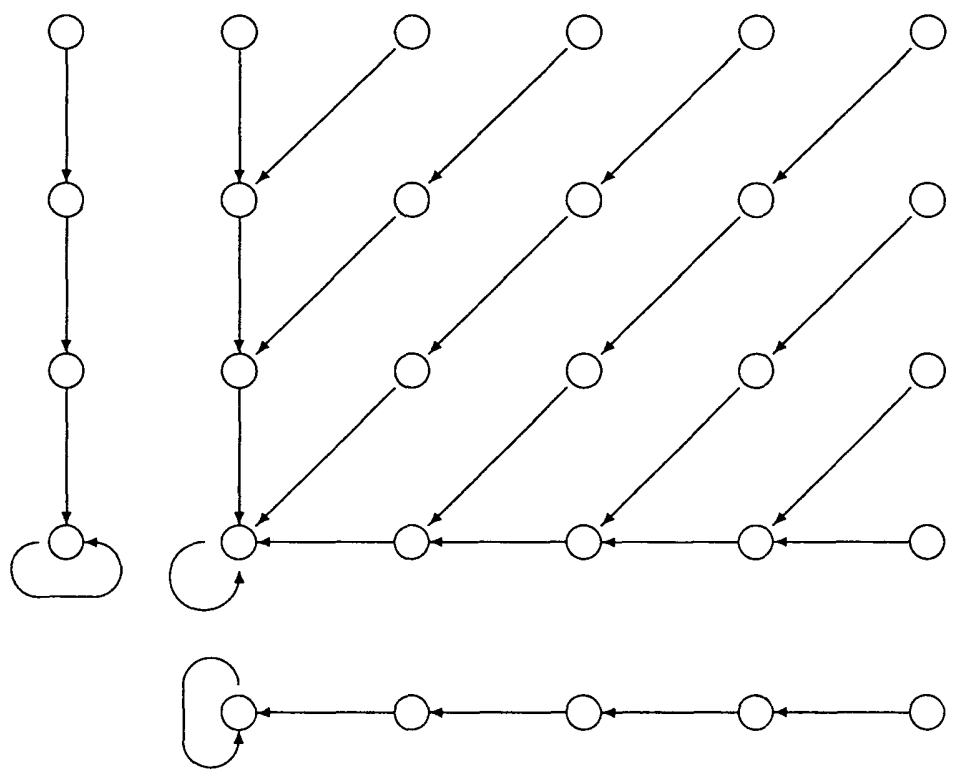

Figure $7:<[2+[1+[1+[1]]]]_{*}>\times<[2+[1+[1]]]_{*}>$

Secondly we consider the product of Figure 4 and 5 . Let $E=<[3+[2]]_{*},[4+$ $[2]+[3]]_{*}>$ (Figure 4) and $F=<[2+[2]]_{*},[3]_{*},[4+[2]]_{*}>$ (Figure 5). Since the limit cycles of $E$ and $F$ are $\langle 0,1\rangle$ and $\langle 0,1,2\rangle$, that of the product of $E$ and $F$ is 
$<(0,0),(1,1),(0,2),(1,0),(0,1),(1,2)>$. So the product of $E$ and $F$ are represented by

$<(E \times F)(0,0),(E \times F)(1,1),(E \times F)(0,2),(E \times F)(1,0),(E \times F)(0,1),(E \times F)(1,2)>$ and each subtrees of $E \times F$ are calculated as follows;

$$
\begin{aligned}
(E \times F)(0,0) & =\left[6+[2] \otimes[2]+[2] \otimes[4+[2]]_{*}+[4+[2]+[3]]_{*} \otimes[2]\right]_{*} \\
& =[6+[4]+2[8]]_{*} \\
(E \times F)(0,1) & =\left[9+[2] \otimes[0]+[2] \otimes[2+[2]]_{*}+[4+[2]+[3]]_{*} \otimes[0]\right]_{*} \\
& =[9+[4]]_{*} \\
(E \times F)(0,2) & =\left[12+[2] \otimes[2]+[2] \otimes[3]_{*}+[4+[2]+[3]]_{*} \otimes[2]\right]_{*} \\
& =[12+[4]+[6]+[8]]_{*} \\
(E \times F)(1,0) & =\left[8+[2] \otimes[2]+[2] \otimes[3]+[2] \otimes[4+[2]]_{*}+[3] \otimes[4+[2]]_{*}+[3+[2]]_{*} \otimes[2]\right]_{*} \\
& =[8+[4]+2[6]+[8]+[12]]_{*} \\
(E \times F)(1,1) & =\left[12+[2] \otimes[2+[2]]_{*}+[3] \otimes[2+[2]]_{*}\right]_{*} \\
& =[12+[4]+[6]]_{*} \\
(E \times F)(1,2) & =\left[16+[2] \otimes[2]+[3] \otimes[2]+[2] \otimes[3]_{*}+[3] \otimes[3]_{*}+[3+[2]]_{*} \otimes[2]\right]_{*} \\
& =[16+[4]+3[6]+[9]]_{*}
\end{aligned}
$$

So the transition diagram of an isomorphic system of the product of Figure 4 and 5 is illustrated as Figure 8.

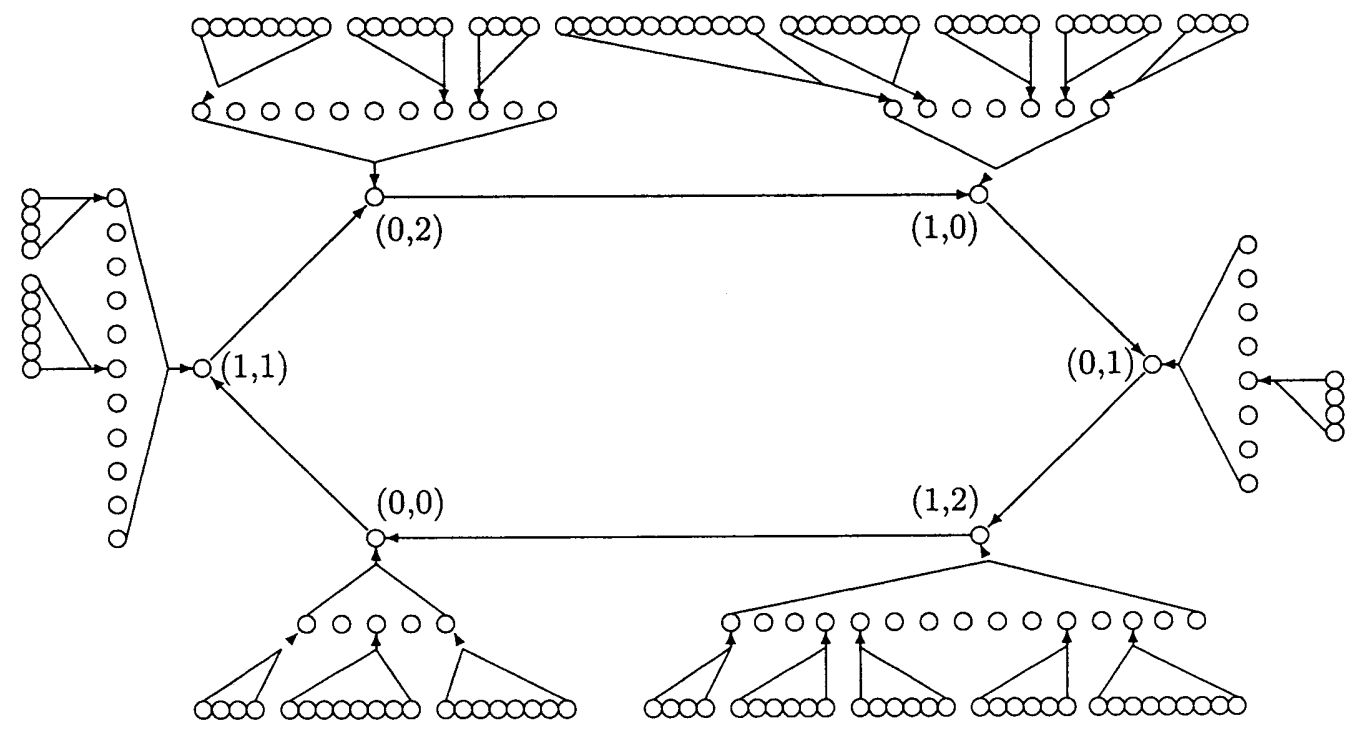

Figure 8: $<[3+[2]]_{*},[4+[2]+[3]]_{*}>\times<[2+[2]]_{*},[3]_{*},[4+[2]]_{*}>$ 


\section{Conclusion}

In this paper we introduced tree expressions which can represent transition diagrams of finite systems, and presented their explicit product formula. Tree expressions can represent transition diagrams of all finite systems, and make us be able to deal with transition diagram of finite systems by algebraic methods. And our product formula is useful for analysis and applications of finite systems which can be separated or is the product of finite systems.

But there exist several weak points. First, tree expressions have redundancy. The larger the number of nodes of a system is, the longer the length of its tree expression. Second, tree expressions are not unique. It is easy to introduce uniqueness of tree expressions by lexicographical order. But the tree expression of the product of normal tree expressions to be got by applying product formula is not always normal, where a tree expression is normal if $\mathrm{t}$-sets in it are in order.

Future works are to overcome these weak points and to apply it to the analysis of cellular automata.

\section{References}

Y. Kawahara (1991). Existence of the characteristic numbers associated with cellular automata with local transition rule 90, Bulletin of Informatics and Cybernetics, 24, 121-136.

H. Y. Lee and Y. Kawahara (1992). On dynamical behaviors of cellular automata CA60, Bulletin of Informatics and Cybernetics, 25, 22-27.

Y. Kawahara, S. Kumamoto, Y. Mizoguchi, M. Nohmi, H. Ohtuka and T. Shoudai (1995). Period lengths of cellular automata on square lattices with rule 90, Journal of Mathematical Physics, 36(3), 1435-1456.

H. Lee and Y. Kawahara (1996). Transition Diagrams of Finite Cellular Automata, Bulletin of Informatics and Cybernetics, 28(1), 47-69.

S. Inokuchi and T. Sato (2000). On Limit Cycles and Transient Lengths of Cellular Automata with Threshold Rules, Bulletin of Informatics and Cybernetics, 32(1), 23-60.

S. Kumamoto and M. Nohmi (in preparation). On Transition Diagrams of Integral Affine Dynamical Systems, in preparation.

Received March 17, 2004

Revised March 29, 2004 